\title{
Consequences of 'conversations not had': insights into failures in communication affecting delays in hospital discharge for older people living with frailty
}

Sabi Redwood, Bethany Simmonds, Fiona Fox, Ali Shaw, Kyra Neubauer, Sarah Purdy \& Helen Baxter

\begin{abstract}
Objectives: Older people living with frailty (OPLWF) are often unable to leave hospital even if they no longer need acute care. The aim of this study was to elicit the views of health care professionals in England on the barriers to effective discharge of OPLWF.

Methods: We conducted semi-structured interviews with hospital-based doctors and nurses with responsibility for discharging OPLWF from one large urban acute care hospital in England. The data were analysed using the constant comparative method.

Results: We conducted interviews with 17 doctors (12 senior doctors or consultants and 5 doctors in training) and six senior nurses. Some of our findings reflect well-known barriers to hospital discharge including service fragmentation, requiring skilled coordination that was often not available due to high volumes of work, and poor communication between staff from different organisations. Participants' accounts also referred to less frequently documented factors that affect decision making and the organisation of patient discharges. These raised uncomfortable emotions and tensions and were often ignored or avoided. One participant referred to 'conversations not had', or failures in communication, because difficult topics about resuscitation, escalation of treatment and end-of-life care for OPLWF were not addressed.
\end{abstract}

Conclusions: The consequences of not initiating important conversations about decisions relating to the end of life are potentially far reaching not only regarding reduced efficiency due to delayed discharges, but also for patients' quality of life and care. As the population of older people is rising, this becomes a key priority for all practitioners in health and social care. Evidence for support for practitioners, OPLWF and their families is needed to ensure these vital conversations take place so that care at the end of life is humane and compassionate. 


\section{Introduction}

Older people living with frailty (OPLWF) have a highly complex range of needs because of increased vulnerability resulting from ageing-associated decline in reserve and function, compromising their ability to cope with every day or acute stressors. ${ }^{1}$ They are often unable to leave hospital even if they no longer need acute care, ${ }^{2}$ with negative consequences for their health, physical function, independence and care needs, ${ }^{3}$ and the wider service, such as staff well-being and morale, interprofessional relationships and service costs. ${ }^{4}$ Many countries have recognised these challenges and introduced initiatives to reduce unnecessary delays, but they continue to rise in England, ${ }^{2-5,6}$ and internationally. ${ }^{7}$ In this study we focus on the National Health Service (NHS) in England. Responsibility for delayed hospital discharges lies with the NHS or local authorities ${ }^{8}$ but service fragmentation has meant that collaborative working to ensure timely discharge for OPLWF has remained challenging. Identified problems include care coordination and information sharing between different parts of the system, aggravated by shortages in staff with appropriate skills, and some hospital staff's lack of knowledge of the needs of OPLWF. ${ }^{3,6,8}$ It remains unclear however how these factors interact to impede effective discharge and exacerbate delays. We sought to elicit the views of health care professionals working in the NHS on the barriers to effective discharge of OPLWF in order to identify and describe some of the less clearly defined problems affecting decision-making and organising the discharges for OPLWF.

\section{Methods}

We conducted a qualitative study ${ }^{9}$ involving semi-structured interviews with hospital-based doctors and nurses with responsibility for discharging OPLWF in one large urban acute care hospital in England. We purposefully sampled a higher proportion of senior doctors because of their leadership role in discharging patients and their influence on team and specialty culture, and on clinical practice. ${ }^{10}$ We invited consultants (senior doctors), doctors in training, and senior nurses from a range of specialities because professional cultures, knowledge and practices can vary, particularly in relation to the care of OPLWF.[11] Interviews were conducted by two experienced researchers and were based on an interview guide exploring participants' experience of caring for OPLWF. Participants were also invited to describe in detail specific anonymised cases of successful and unsuccessful discharges. 
Data collection took place over six months in 2017. Interviews were recorded, transcribed verbatim, anonymised and assigned a participant number.

Data were analysed using the constant comparative method, based on Charmaz' constructivist grounded theory approach. ${ }^{12}$ This involved an inductive and iterative process of close reading of the data, coding, constant comparison and elaboration of initially constructed themes, ${ }^{13}$ using the QRS NVivo data management software.${ }^{14}$ Codes and categories were discussed and reviewed regularly by the author team comprising clinicians and social scientists. Categories of data and thematic relationships were then identified and written up as descriptive and interpretive accounts. As this was an exploratory study, we did not plan to arrive at a complete description of all aspects affecting discharge delays, but collected data to provide new insights into current understandings.

\section{Results}

A total of 23 interviews were conducted: 17 participants were doctors ( 12 senior doctors or consultants and 5 doctors in training) and 6 were senior nurses. Eleven worked in the care of older people (COP), and 12 worked in a range of specialities including respiratory medicine (3), neurology (1), cardiology (2), surgery (2), renal (1), palliative care (2), emergency department (ED, 1) and medical admissions unit (MAU, 1).

Some of our findings resonate with existing evidence and reflect well-known barriers to the discharge of OPLWF. ${ }^{15,16}$ For example, participants referred to the fragmentation of services requiring skilled coordination that was often not available due to high volumes of work; poor communication between staff from different organisations and professional backgrounds; and waiting for staff from other organisations to carry out tasks. This included completing paperwork, the time it takes for families to make decisions, sourcing social care packages or placements, organising medications to take home and arranging suitable transport. While the data relating to these factors were rich and detailed, recent research has already provided insights into how they relate to patient safety and delays in hospital discharges. ${ }^{17,18}$

The focus of this paper is participants' accounts of less openly acknowledged factors affecting decision-making and discharge coordination. One of the participants referred to 'conversations not had' to describe how a lack of dialogue with patients, families, carers and 
other professionals, and failures of communication in the care coordination processes had harmful consequences for patients in relation to their health outcomes and quality of life, and the quality and safety of their care. Other consequences involved admission to hospital when patients may have been managed more appropriately in their own homes or in another care setting; inappropriate investigations and treatments; and prolonged stays in hospital. The reasons for conversations not taking place varied. Participants' accounts indicated that they or others may be feeling inadequately prepared for conversations in an environment that is considered unsuitable for eliciting sensitive information and conducting potentially complex discussions.

Unlike concerns about care coordination across health and social care, which are usually openly discussed among colleagues and are part of the public discourse about delayed discharges, there were other factors which raised uncomfortable emotions and tensions and were often ignored or avoided. Participants suggested that important conversations were not initiated about end-of-life care, treatment limits and whether resuscitation in the event of cardiac or pulmonary arrest should be attempted. The topic of death and dying was also often not raised by clinicians, and participants reported that patients and families did not ask questions.

Our analysis coalesced around two themes 'uncertainty compounded by lack of information' and 'the hospital as an unsuitable place for end-of-life conversations and care'. These are developed in the following sections with illustrative interview excerpts from participants. A table which summarises the themes, constituent categories and further interview excerpts is provided in the online supplement.

\section{Uncertainty compounded by lack of information}

Participants described OPLWF as clinically complex and the course of their often multiple conditions unpredictable. A challenge identified by several participants was the uncertainty about when someone was approaching the end of life, as well as the complexity of clinical decision making and care planning for patients whose conditions fluctuated. Participants reported how this often led to clinical investigations being ordered and treatments being started when this may not be the best course of action. 
As a profession as a whole, the default setting for medics is to do things and it is going to take a bit of time to work out actually not doing things is equally good.

Participant 11, Consultant, MAU

Some also commented on the ethos of hospital-based medical practice, which was mostly about diagnosis and treatment to preserve life. The reluctance to let go of active interventions and instead have important conversations with patients was highlighted:

The main thing is that you have the conversations [about end of life] and I think there is a real problem with our health system that the default is to treat, and these conversations are not had.

Participant 7, Consultant, COP

The initiation of investigations and interventions was reported to lead to delays because of waits required to carry out tests and get results, even if treatment options were limited or absent. In case of invasive procedures, this could lead to a deterioration in a patient's condition.

The clinical uncertainty was often compounded by a lack of what some participants referred to as 'collateral information' about a patient's usual functioning and abilities, their social circumstances, and a history of events leading to a decision to access hospital care. This information is needed to complement the clinical picture and medical history but could only be obtained from someone who knows the patient well, such as a relative, closely involved in the patient's life, or a professional carer. It was seen to be especially important when a patient was assessed at the emergency department:

That type of information is really useful for us, because then we know as clinicians how much we need to investigate acutely, what's caused the admission, or whether actually a number of factors are already in the history that clearly show why the patient's ended up in hospital, and then it speeds up the process of investigation and discharging the patient, definitely. That collateral information is vital.

Participant 6, Consultant, ED 
Nurses also considered such information as essential for care and discharge planning. This participant described the difficulty of accessing it in the context of increasing demand and patient numbers:

It's getting the right information to make the right plan. (...) Everybody should be able to take a history from patients, but it's probably not done in the right way. There's not time. If AMU [Acute Medical Unit] are turning out 200 patients a day, the doctors aren't going to be able to speak to 200 relatives.

Participant 22, Senior Nurse, COP

Participants consistently reported how the lack of this information undermined safe and patient-centred decision making, often leading to inappropriate admission to hospital resulting in further functional decline. Even if it was collected close to patients' arrival at the hospital, once they were admitted to a ward, this 'collateral information' was at risk of being lost. Participants' account pointed to several reasons for this: collecting and documenting this information could not be prioritised over urgent clinical work; it was not valued in the same way as information from clinical investigations and considered a relatively low status task compared to clinical work. However, using and integrating 'collateral information' into medical decision making was considered an important part of the discharge process as this consultant explained:

You need to pool that information, combine it with the medical information, coordinate that and make sure you've got a safe discharge and you know that would work.

Participant 7, Consultant, COP

Despite its crucial role in the care planning and discharge process, participants reported that it was often not available, leading to delays in discharge.

\section{The hospital as an unsuitable place for end-of-life conversations and care}

There was consensus among professionals who were not specialists in care of OPLWF that the acute hospital was an unsuitable environment to talk to patients and families about end-of-life decisions. One reason given was the pressure hospital staff were under: 
End of life care is not usually our conversation. (...) Those conversations are quite difficult (...) I'm sure we've all been in with somebody who's at the end of life (...) but I don't think we've actually sat down (...) with a patient talking about where they want to be, what treatment they want. It sounds awful but we haven't got the time for that.

Participant 2, Senior Nurse, cardiology

The acute care environment such as the emergency department and medical admissions unit were seen a stressful and distressing for patients. But if such conversations had not taken place previously, they needed to take place now:

There are clearly patients who should not be resuscitated, and it's a difficult discussion to have in the cold light of day, but it should be had, and it doesn't. And then actually it's a very poor experience for people when it has to be done in here [MAU].

Participant 19, Consultant, MAU

Participants highlighted other negative consequences of lack of (information on) end-of-life decisions. The excerpt below relates to the obligation on staff to follow resuscitation protocols in the event of a patient's respiratory and/or cardiac arrest, even when it was clear that the patient was not going to benefit for further treatment:

If the conversation hasn't happened, and the patient has collapsed, they are not breathing, we've got to start resuscitation procedures.

Participant 4, Senior nurse, COP

In the same way that nurses had to initiate resuscitation procedures, doctors in training who provide first-line medical care in the hospital setting followed protocols for diagnosis and treatment. This was seen to be particularly challenging in emergency situations, such as when a OPLWF is in crisis and has been conveyed to the ED. In this pressurised environment, which requires decisions to be taken quickly, difficult conversations about the appropriateness of treatment were not considered an option; active treatment was seen to present a less problematic path: 
The junior doctor who (...) sees the ninety year old coming in [via the ED], struggling to breathe with a nasty chest infection has only so much time, so the easiest thing to do is to start them on antibiotics, start the oxygen and then they get passed on to MAU where the junior doctor there says okay (...) I'll do the blood culture, I'll do this, that, and then pass the patient on. Because the conversations take a lot longer and if done well, you need to have the time. (...) Typically, it's quicker to do things and not have the talk, because everything has to happen quickly, you have got to get people out otherwise you hit the [waiting time] targets. (...) So, there are perverse incentives that sometimes stop us doing the right thing.

Participant 11, Consultant, MAU

Even when such patients are seen by senior doctors, participants' accounts indicated that the acute care setting was not the right place for conversations about advance care planning:

We think these [end-of-life and resuscitation conversations] should be happening on an outpatient basis with our colleagues for patients with chronic illness or with their general practitioners, ideally. (...) I think it would be better had they happened previously when the patient was well but had obvious multiple chronic conditions that meant that cardiopulmonary resuscitation was not going to be appropriate.

Participant 6, Consultant, ED Similarly, it was suggested that while patients' closeness to death often remains uncertain, clinical deterioration in those with multiple morbidities tends to follow a predictable path, providing opportunities to discuss end-of-life decisions before they arrive in hospital in crisis:

There are an awful lot of people with long-term conditions where there's a relatively predictable trajectory to their illness. They're going to progress to heart failure or renal failure, or respiratory failure and it's never discussed with people until the crisis and that's not good medicine. It's not kind medicine.

Participant 23, Consultant, respiratory medicine 
However, even if such conversations had taken place and decisions had been made, the processes for documenting them so that they can be accessed at the right time by other professionals were not deemed reliable. Participants expressed frustration at information systems' designs which did not consistently allow access to such vital information.

Some participants' accounts suggested that 'conversations not had' related not only to endof-life and resuscitation decisions, but also to plans about future treatments or relief of distressing symptoms in the event of patients' sudden deterioration. Such lack of planning can often lead to patients being admitted to hospital unneccessarily:

There is an issue with care homes and [general practitioners] so that the default is to send patients in to hospital, so if we know we are not going to operate on somebody and they have got terminal cancer and that we know that their bowel might block off, if their bowel blocks off and they are in a care home, they will be sent to hospital even though there's nothing we can do about it.

Participant 15, Consultant, surgery

Other reasons for inappropriate admissions such as the one just described related to the absence of advanced care planning by primary care and care home staff; distressing symptoms experienced by people at the end of life which had not been anticipated, resulting in carers contacting the ambulance service; and a lack of adequate services to respond to the clinical needs of patients at the end of life in the community out of hours. Participants' accounts described a range of circumstances triggered by a lack of advanced care planning that resulted in futile resuscitation attempts; admissions to intensive care units that led to long stays in hospital and poor quality of life in patients' final days and weeks; and unrealistic expectations by patients and families about the success of cardiopulmonary resuscitation.

The consequence of these types admissions was characterised by this participant:

It's the worst thing for someone who's old and frail to be whisked into an acute building away from people who've been looking after them, people they know, to die alone in hospital. It's not kind.

Participant 23, Consultant, respiratory medicine 
Many participants expressed similar thoughts about how current practice was lacking humanity and voiced regrets about their inability to better support dying patients.

\section{Discussion}

The findings of this exploratory study of reasons for delays in the discharge from hospital of OPLWF resonate with existing evidence while also identifying less frequently acknowledged factors affecting discharges, in particular those relating to the uncertainty about when a frail older person is approaching death.

Such uncertainty can produce anxiety, especially for less experienced staff, which can lead to feelings of vulnerability and the avoidance of decision making ${ }^{19}$. More experienced staff have been shown to be able to tolerate uncertainty and to prefer less risk-averse patient management. ${ }^{20}$

A strategy to reduce uncertainty is to seek information either in the form of clinical information through investigations and tests, or information about patients' social background and functional status. While the former was described by participants as a frequently used strategy which led to delays in decision making, the latter was often frustrated by a lack of time to access or retrieve it.

The acute hospital environment was perceived as unsuitable for raising topics about end-oflife decisions with OPLWF because they were likely to elicit uncomfortable emotions for patients and their families. Several participants suggested that such conversations should be initiated by staff in primary or community care rather than by clinicians in the acute hospital setting, whose primary task is to provide short episodes of clinical care, diagnostics and curative services in response to urgent threats to health. Therefore, it may be difficult for many clinicians to find the right time or the right way to initiate conversations with patients about decisions they fear they may not be ready for and which they see as intrinsically negative. This may be because 'do not attempt cardiopulmonary resuscitation' (DNACPR) orders are commonly misinterpreted to mean that all care should be reduced, raising concerns that patients may be receiving substandard care. There is evidence to suggest that this is indeed the case. ${ }^{21-23}$.

An OPLWF's arrival at the acute hospital was seen as the start of an almost inevitable process of medical interventions, described as 'unkind medicine' by one participant, where 
the environment was seen to be unsuitable for their needs and lacked appropriately prepared staff to provide high quality and safe care for those at the end of life as a result of frailty. Yet paradoxically, hospitals are considered a place of safety when other services have been unable to respond in a crisis, fuelling demand on hospital services. ${ }^{2}$ The current health and social care system is ill-equipped to receive OPLWF who have many complex health and social needs back into community settings so that these patients must remain in acute hospitals, increasing their vulnerability, the risk of functional decline and greater care needs after discharge, as well as the risk of emergency re-admission. ${ }^{2,4}$

This was an exploratory study in one large urban acute care hospital in England. We focused on mostly senior clinicians because they lead clinical teams and decision making on discharge, and their behaviour guides that of doctors in training and other professionals. While it was important to understand their perspective, we were unable to include the views of patients and carers, therapists, social workers and discharge coordinators. We were thus unable to explore the wider context shaping practices around discharge. However, this study generated insights into problems that are less well acknowledged and have hitherto not been reported in the context of delayed hospital discharges. These difficulties were linked to 'conversations not had' about resuscitation and end of life decisions, as well as patients' personal, social and family circumstances. Our research team comprised clinical and non-clinical members and met regularly to debrief the researchers who carried out interviews. The team scrutinised the data and nascent analysis to enable them to be open to developing interpretations and conscious of constructing familiar assumptions. ${ }^{12}$

The reluctance among patients and doctors to discuss resuscitation and treatment plans for the end-of-life are well documented. ${ }^{24}$ In the absence of conversations about life-sustaining treatment, this cycle of silence on issues of death and dying will persist, fuelled by multiple barriers, such as clinical uncertainties, fear of the impact on patients, navigating patient readiness, and feeling apprehensive about such discussions because of a lack of training or inadequate preparation. ${ }^{25}$ There are metrics regularly collected on a range of problems in the care process that lead to delays in discharge from hospital for OPLWF, it is difficult to assess the scale of delays that are due to 'conversations not had'. However, their consequences are potentially far reaching, not only regarding reduced efficiency, concerns about patient safety and quality of care, and the perpetually increasing numbers of days 
'lost' to delayed discharges, but also for patients' quality of life and their experiences at the end of life. There are substantial gaps in the evidence about the skills needed for effective, sensitive communication with patients about end of life decisions. ${ }^{26,27}$ Frailty and clinical uncertainties compound the problem for clinicians in initiating these conversations. However, patients understand the need for and are willing to have them. ${ }^{28}$ Clinicians working in the COP who participated in our study reported that these conversations were part of their daily practice. However, discussing and planning end of life care should not remain the exclusive skill set for those working in relevant specialist areas. As the population of OPLWF is rising, this will become a key priority for all practitioners in health and social care. However, existing evidence about how professionals can be supported to carry out these conversations in community, primary care and hospital settings remains scarce. Similarly, further research is needed to provide OPLWF and their families with information and support to feel confident in talking about humane and compassionate care at the end of life, avoiding unnecessary investigations and futile treatments. 


\section{References}

1. Chen X, Mao G and Leng SX. Frailty syndrome: an overview. Clin Interv in Aging 2014; 9: 433-41.

2. National Audit Office. Discharging older patients from hospital. https://www.nao.org.uk/report/discharging-older-patients-from-hospital/ (2016, accessed 18 November 2019).

3. Glasby J, Littlechild R, Pryce K. All dressed up but nowhere to go? Delayed hospital discharges and older people. J Health Serv Res Policy 2006; 11 (1): 52-8.

4. Rojas-García A, Turner S, Pizzo E, Hudson E, Thomas J and Raine R. Impact and experiences of delayed discharge: A mixed-studies systematic review. Health Expect 2018; 21 (1): 41-56.

5. National Audit Office. Health and Social Care Integration. https://www.nao.org.uk/wpcontent/uploads/2017/02/Health-and-social-care-integration.pdf (2017, accessed 18 November 2019)

6. Glasby J. The holy grail of health and social care integration. BMJ 2017; j801.

7. Theou O, Squires E, Mallery K, Lee JS, Fay S, Goldstein J, Armstrong JJ and Rockwood K. What do we know about frailty in the acute care setting? A scoping review. BMC Geriatr 2018; 18 (1): 139.

8. Humphries R, Hall P, Charles A, Thorlby R, and Hunter H. Social Care for Older People: Home truths. Report for the Kings Fund.

https://www.kingsfund.org.uk/publications/social-care-older-people (2016, accessed 18 November 2019)

9. Tong A, Sainsbury P and Craig J. Consolidated criteria for reporting qualitative research (COREQ): a 32-item checklist for interviews and focus groups. Int J Qual Health Care 2007; 19 (6): 349.

10. Laugaland K, Aase K, Waring J. Hospital discharge of the elderly-an observational case study of functions, variability and performance-shaping factors. BMC Health Ser Res 2014; 14 (1): 365. 
11. Waring J, Marshall F, Bishop S, Sahota O, Walker M, Currie G, et al. An ethnographic study of knowledge sharing across the boundaries between care processes, services and organisations: the contributions to 'safe' hospital discharge. Health Services and Delivery Research (NIHR Journals Library) 2014; 2 (29): 1-160.

12. Charmaz K. The Power of Constructivist Grounded Theory for Critical Inquiry. Qual Inq 2017; 23 (1): 34-45.

13. Charmaz K. Constructing grounded theory: a practical guide through qualitative analysis. London: Sage; 2006.

14. NVivo qualitative data analysis Software; QSR International Pty Ltd. Version 10, 2014.

15. Bauer M, Fritzgerald L, Haesler E, Manfrin M. Hospital discharge planning for frail older people and their family. Are we delivering best practice? A review of the evidence. J Clin Nurs 2009; 18: 2539-2546.

16. Corrigan O, Georgiades A, Davies A, Lane P, Milne E, Speed E and Wood D. Insights into Hospital Discharge - a study of patient, carer and staff experience at Broomfield Hospital. https://www.healthwatchessex.org.uk/wp-content/uploads/2016/06/Insightsinto-Hospital-Discharge-at-Broomfield-Hospital-Full-Report.pdf (2016, accessed 18 November 2019)

17. Baillie L, Gallini A, Corser R, Elworthy G, Scotcher A and Barrand A. Care transitions for frail, older people from acute hospital wards within an integrated healthcare system in England: a qualitative case study. Int J Integr Care 2014; 14(1).

18. Pinkney J, Rance S, Benger J, Brant H, Joel-Edgar S, Swancutt D, Westlake D, Pearson M, Thomas D, Holme I and Endacott R. How can frontline expertise and new models of care best contribute to safely reducing avoidable acute admissions? A mixed-methods study of four acute hospitals. Health Serv Deliv Res 2016; 4 (3).

19. Hillen MA, Gutheil CM, Strout TD, Smets EM and Han PK. Tolerance of uncertainty: Conceptual analysis, integrative model, and implications for healthcare. Soc Sci Med 2017; 180: 62-75.

20. Lawton R, Robinson O, Harrison R, Mason S, Conner M. and Wilson, B. Are more experienced clinicians better able to tolerate uncertainty and manage risks? A vignette 
study of doctors in three NHS emergency departments in England. BMJ Qual Saf 2019; 28 (5): 382-388.

21. Mockford C, Fritz Z, George R, Court R, Grove A, Clarke B, Field R and Perkins GD. Do not attempt cardiopulmonary resuscitation (DNACPR) orders: A systematic review of the barriers and facilitators of decision-making and implementation. Resuscitation 2015; 88: 99-113.

22. Myint PK, Miles, S, Halliday D A and Bowker L K. Experiences and views of specialist registrars in geriatric medicine on 'do not attempt resuscitation' decisions: a sea of uncertainty? QJM Int J Med 2006; 99: 691-700.

23. Fritz Z, Fuld J, Haydock S, Palmer C. Interpretation and intent: a study of the (mis)understanding of DNAR orders in a teaching hospital. Resuscitation 2010; 81: 113841.

24. Myint PK, Rivas CA, Bowker LK. In-hospital cardiopulmonary resuscitation: Trainees' worst and most memorable experiences. QJM Int J Med 2010; 103: 865-73.

25. Brighton $\amalg$ and Bristowe K. Communication in palliative care: talking about the end of life, before the end of life. Postgraduate Med J 2016; 92 (1090): 466-70.

26. Gjerberg E, Lillemoen L, Førde R, Pedersen R. End-of-life care communications and shared decision-making in Norwegian nursing homes - experiences and perspectives of patients and relatives. BMC Geriatr 2015; 15 (1): 103.

27. Parry R, Land V, Seymour J. How to communicate with patients about future illness progression and end of life: a systematic review. BMJ Support Palliat Care 2014; 4 (4): 331-41.

28. Broadhurst HL, Droney J, Callender T, Shaw A, Riley J. Advanced care planning: the impact of Ceiling of Treatment plans in patients with Coordinate My Care. BMJ Support Palliat Care 2018; 9 (3): 267-270.

29.

\section{Declarations}

Ethics approval and consent to participate 
This project received ethical approval from the University of Bristol's Faculty of Medicine and Dentistry Research Ethics Committee (application ID, 19001). All participants provided consent to be interviewed and audio recorded.

Consent for publication

Not applicable.

\section{Availability of data and material}

Given the relatively small number of participants in one NHS Trust, their data contains a lot of contextual information which is likely to lead to the identification of individuals.

Therefore, to protect the participants' anonymity, the study data will not be made available.

\section{Competing interests}

The authors declare that they have no competing interests.

\section{Funding}

The research was supported by the Bristol, North Somerset and South Gloucestershire Clinical Commissioning Group and the National Institute for Health Research (NIHR) Collaboration for Leadership in Applied Health Research and Care West (CLAHRC West) at University Hospitals Bristol NHS Foundation Trust. The views expressed are those of the authors and not necessarily those of the NHS, the NIHR or the Department of Health and Social Care.

\section{Authors' contributions}

SP, HB, KN and SR developed and designed the study. BS and FF collected the data. Analysis was carried out by BS, FF and SR and supported by all authors. SR and BS drafted the manuscript and all authors contributed to the final version.

\section{Acknowledgements}

We thank all our participants who took the time to be interviewed for this study and shared their experiences with us. We also thank Dr Peter Goyder for his assistance with study design and advice on the wider urgent care system. 